\title{
Air Convection Noise of Pencil-beam Interferometer for Long Trace Profiler
}

\author{
Valeriy V. Yashchuk*a, Steve C. Irick ${ }^{\mathrm{a}}$, Alastair A. MacDowell ${ }^{\mathrm{a}}$, \\ Wayne R. McKinney ${ }^{\mathrm{a}}$, Peter Z. Takacs \\ ${ }^{a}$ Lawrence Berkeley National Laboratory, Berkeley, California, 94720 \\ ${ }^{b}$ Brookhaven National Laboratory, Upton, New York, 11973
}

\begin{abstract}
In this work, we investigate the effect of air convection on laser-beam pointing noise essential for the long trace profiler (LTP). We describe this pointing error with noise power density (NPD) frequency distributions. It is shown that the NPD spectra due to air convection have a very characteristic form. In the range of frequencies from $\sim 0.05 \mathrm{~Hz}$ to $\sim 0.5 \mathrm{~Hz}$, the spectra can be modeled with an inverse-power-law function. Depending on the intensity of air convection that is controlled with a resistive heater of 100 to $150 \mathrm{~mW}$ along a one-meter-long optical path, the power index lies between 2 and 3 at an overall rms noise of $\sim 0.5$ to 1 microradian. The efficiency of suppression of the convection noise by blowing air across the beam optical path is also discussed. Air-blowing leads to a white-noise-like spectrum. Air blowing was applied to the reference channel of an LTP allowing demonstration of the contribution of air convection noise to the LTP reference beam. The ability to change (with the blowing technique presented) the spectral characteristics of the beam pointing noise due to air convection allows one to investigate the contribution of the convection effect, and thus make corrections to the power spectral density spectra measured with the LTP.
\end{abstract}

Keywords: pencil-beam interferometer, long trace profiler, air convection, laser beam, pointing stability, X-ray optics, optical metrology, error reduction

\section{INTRODUCTION}

The long trace profiler (LTP) is the basic metrology tool for testing the X-ray optic figure with slope variation on the order of one microradian. ${ }^{1-11}$ However, the task of designing high performance X-ray optical systems suitable for submicron focusing requires improvement of dedicated metrology instrumentation to sub-microradian accuracy of lowspatial-frequency slope measurements. All elements of the instrument must be free of any systematic error and noise has to be suppressed to at least this level. In particular, in the case of a pencil beam interferometer in a LTP, this requires a corresponding pointing stability of the light beam. The pointing instability of a LTP light source directly contributes to the slope measurement error. In order to quantify pointing instability, an LTP with an additional reference beam has been developed. ${ }^{5,6}$ In the optical schematic of this instrument, the reference slope signal from a stationary reference mirror is recorded simultaneously with the slope trace measurement of a mirror under investigation. Then, the reference trace is subtracted from the sample trace, providing data basically free of error due to pointing instability. A similar approach has been used in a Dual-beam laser deflection sensor described in Ref. ${ }^{12}$ Unfortunately, compensation of the laser pointing instability with a reference beam has some limitations. The optical paths for the reference and the sample beams are significantly different. Moreover, the optical path for the reference beam is subject to change during the LTP measurement. As a result, the noise and systematic errors due to the imperfections of the LTP optics and convection of air along the optical paths are different for the reference and sample beams and can not be completely eliminated with the subtraction of the reference signal from the sample signal. Therefore, rigorous information on the laser pointing stability, on the effects which lead to increase the pointing variation, and on method to decrease the variations is especially important.

The pointing instability of a laser is determined as a temporal angular drift of the beam direction - Fig. 1a. In order to specify the pointing instability of a laser, the usual practice is to provide a value of the change of the beam direction upon a certain change of the ambient temperature in the units of $\mathrm{rad} /{ }^{\circ} \mathrm{C}$. Such a specification of the pointing stability does not provide the laser user with adequate information on the temporal beam direction stability one can achieve.

*vvyashchuk@lbl.gov; phone +1 510-495-2592; fax +1 510-486-7696 
Here, we are using a presentation of the pointing stability via an angular noise power density (NPD) spectrum, similar to a noise power density spectrum ${ }^{13,14}$ usually used in electronics ${ }^{15}$ or a power spectral density (PSD) distribution applied to surface roughness measurements. ${ }^{16-19}$

Figure 1a shows the temporal dependence of laser beam position deviation (in the horizontal direction) from the averaged position measured with a beam profiler ${ }^{20}$ and a fiber coupled diode laser module ${ }^{21}$ placed at a distance of $10 \mathrm{~cm}$ from the profiler. At the sampling time of $0.1 \mathrm{sec}$ (Fig. 1a), the variation (rms) of the beam position was found to be $\sigma_{x} \approx 0.26 \mu \mathrm{m}$. The variation magnitude is in a good agreement with the laser pointing stability of about $0.1 \mu \mathrm{rad}$ and the profiler positioning accuracy of about $0.1 \mu \mathrm{m}$ specified for the instruments.

In order to build an NPD spectrum from a discrete temporal sequence we use a standard procedure. The measured temporal sequence denoted as $P_{r}\left(t_{r}\right)$ of $N$ points total is transformed into a noise power density spectrum $D_{s}\left(f_{s}\right)$ via the discrete Fourier transformation:

$$
D_{s}\left(f_{s}\right)=\Delta t \cdot \mid \text { Fourier }\left.\left(P_{r}\left(t_{r}\right)\right)\right|^{2}=\frac{\Delta t}{N} \sum_{r=1}^{N} P_{r}\left(t_{r}\right) \cdot \exp \{2 \pi i(r-1)(s-1) / N\},
$$

where the same definition of discrete Fourier transformation as in Mathematica ${ }^{\mathrm{TM}}$ is used.

The result of application of the transformation (1) to the data for the temporal position noise in Fig. 1a is shown in Fig. 1b. Due to the limit of the length of the temporal sequence measured, there is a large spread of data at higher frequencies. The limited sequence can be thought as a product of an unlimited sequence and a rectangular temporal window. Fourier transformation of a rectangular window gives the high frequency variations of a resulted noise power spectrum. ${ }^{13}$ One of the common ways to decrease the variations is to use a specially shaped window with smooth sides. This approach has a disadvantage of arbitrariness of the window shape. There are dozens of windows commonly used and none of them has any physical reason for priority (see also discussion in Ref. ${ }^{22}$ ). The amplitude of the variations can be statistically decreased by splitting an initial temporal sequence into two shorter sequences and averaging over two noise power spectra for each of the sequences. The efficiency of the procedure is illustrated in Fig. 1c. The cost of the averaging is the loss of the lowest frequency point of the initial spectrum. The initial sequence can be divided to a larger number of subsequences providing more efficient averaging and leading to the loss of lower frequency points of the NPD spectra.

Even stronger suppression of the high frequency variations can be obtained with a simple procedure described in Ref. ${ }^{22}$. The technique is to partition the initial measured data into $K$ subsequences, each of $M$ consecutive measured points. The subsequences can overlap and, in this sense, they are not independent. Each subsequence is separately transformed into a NPD spectrum (1). Finally, the $K$ spectra are averaged at each frequency to produce a spectrum with a reduction of the spread of high frequency data by factor of $\sqrt{K}$.
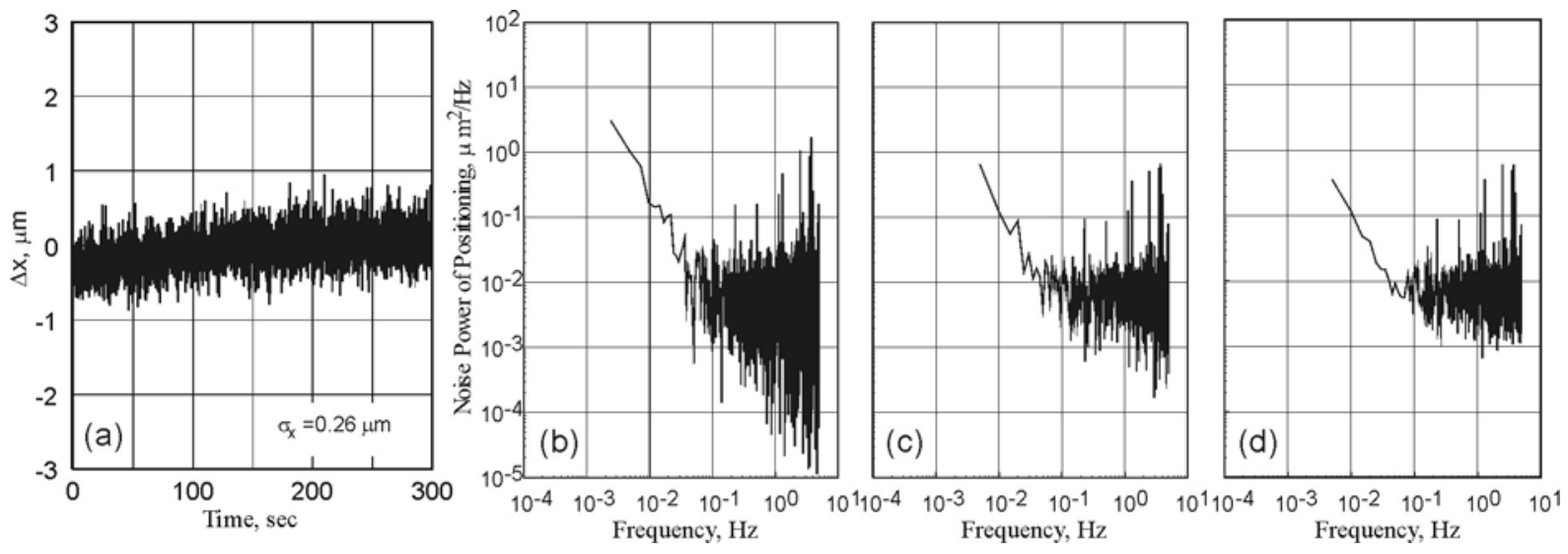

Figure 1: (a) Temporal dependence of the laser beam position deviation from the averaged position measured with a beam profiler (NanoScan ${ }^{\mathrm{TM}}$ High-Power Sub-Micron Accuracy Beam Profiler ${ }^{19}$ with $3.5 \mathrm{~mm}$ aperture) and a fiber coupled diode laser (57 ICS-Series, ${ }^{20} \lambda=670 \mathrm{~nm}, 3 \mathrm{~mW}$ light power) placed on the distance of $10 \mathrm{~cm}$ from the profiler. The profiler sampling time was $0.1 \mathrm{sec}$; (b) NPD spectrum obtained via discrete Fourier transformation (1) of the temporal dependence shown in Fig.1a; (c) NPD spectrum averaged over two spectra obtained by splitting the temporal dependence in Fig. 1a; (d) NPD spectra obtained with multiple averaging over shifted spectra (see text for details). 


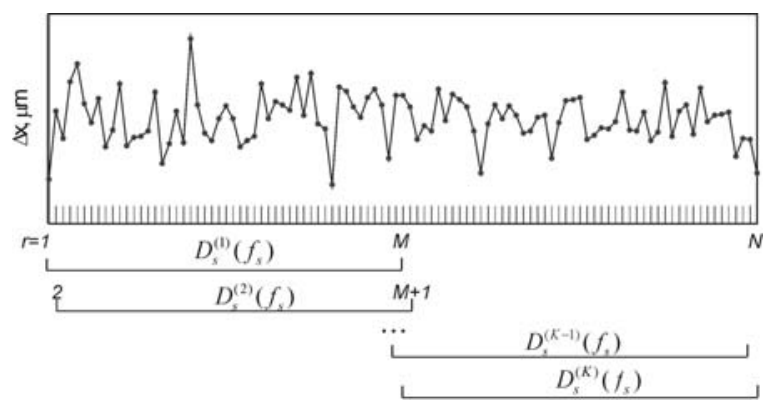

Figure 2: Illustration of the averaging procedure with partitioning into multiple dependent subsequences (see text for details).

The described procedure with the parameters $M=K=N / 2$, where $N \approx 4000$ is the length of the initial sequence that was applied to the measurement shown in Fig. 1a and transformed into the NPD spectrum presented in Fig. 1d. At the chosen parameters, the original data are partitioned into a set of sub-sequences sequentially shifted by one point as illustrated by Fig. 2. Note that in Fig. 1d the resulting variance at higher frequencies is even smaller than it is after averaging over two separated subsequences - Fig. 1c. The averaging procedure described above was applied to the all data shown throughout this work.

In Sec. 2, we investigate the effect of air convection on laser-beam pointing stability. The data of using air blowing as a method to suppress low-frequency part of the beam-pointing noise due to air convection are presented in Sec. 3. The effects of air convection on slope measurements with the LTP II are discussed in Sec. 4 and Sec. 5.

\section{EFFECT OF AIR CONVECTION ON LASER POINTING STABILITY}

Pointing stability was measured by observing the temporal dependence of a laser beam position detected with accuracy of $\leq 0.1 \mu \mathrm{m} / \sqrt{\mathrm{Hz}}$ (compare with data in Fig. 1) with the profiler placed at a known distance from the laser. At the baseline of the set-up of about $1 \mathrm{~m}$, the sensitivity to variation of the beam direction is $\sim 0.1 \mu \mathrm{rad} / \sqrt{\mathrm{Hz}}$. In order to stimulate air convection, a heater was placed under the light-beam path. The heater was made of one rectangular loop, 12-in $\times 2$-in, of stainless steel wire (0.009-in diam.) that has a resistance of $17.4 \mathrm{Ohm}$ at room temperature. For the experiments with the optical path shielded against air-convection perturbations, a one meter long stainless steel tube was placed to enclose the beam. The tube had a $3 / 4$-in outer diameter and 0.04 -in wall thickness. The experimental set-up was assembled on an optical bench placed on a vibration-insolated optical table inside a hutch. An air conditioner kept the laboratory room temperature stable within $\pm 0.1^{\circ} \mathrm{K}$ for a period of $\sim 1$ hour. Temperature variations inside the closed hutch were about $\pm 20 \mathrm{mK}$.

Figure 3 shows temporal dependences of the beam position deviation from the averaged position in the horizontal, $\mathrm{x}$, direction (Fig. 3a) and in the vertical, y, direction (Fig. 3b). In the course of all these measurements, the heater was installed and supplied at the power indicated in each plot. The traces corresponding to the shielding tube in place, surrounding the beam, were recorded at the heater power of $170 \mathrm{~mW}$. That was the maximum power applied in these experiments.

Figure 4 presents the NPD spectra calculated from the temporal data shown in Fig. 3. The lower frequency increase seen on the spectra obtained when the shielding tube was removed can be thought as an indication of perturbation of the lightbeam optical path due to air convection inside the hutch. The observation that at larger, $170 \mathrm{~mW}$, power, the perturbation is larger in the vertical direction also agrees with the hypothesis about a convection effect. Indeed, the steel top of the optical bench used for the set-up assembly serves to flatten the air temperature distribution inside the closed hutch in the horizontal direction with a maximum temperature gradient to be directed vertically. Therefore, at such a temperature distribution, one should expect the air convection to be directed vertically, rather than horizontally.

A visualization of a flattened convection field with a laser holographic interferogram can be found in Ref. ${ }^{23}$ In this case, the air is heated with a horizontal flat plate. The resulted convection field induces a system of almost horizontal interferometric fringes illuminating a variation of air refractive index due to the change of density of the air. Correspondingly, the effective length of the horizontal optical path mostly varies in the vertical direction.

In our case of the wire-loop-heater and at relatively small heat power applied, the convection field is unstable due to comparable environmental perturbations that affect the optical path also in horizontal direction. The perturbations lead to a deliquescent convection flow resulting in a pointing instability almost equal in the vertical and in the horizontal directions. 

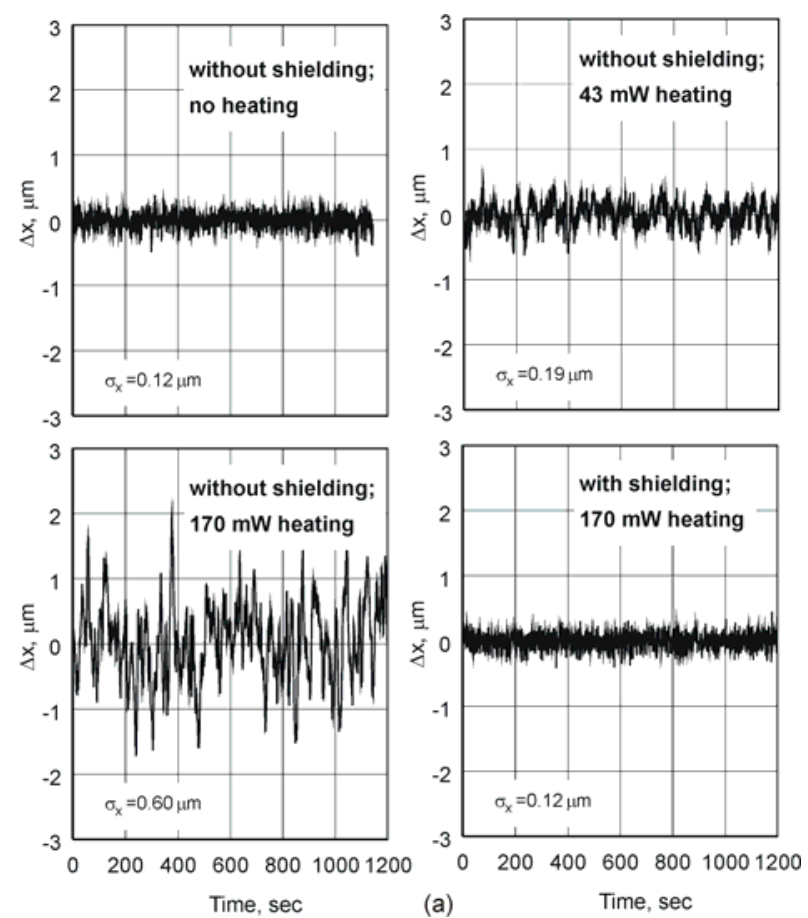
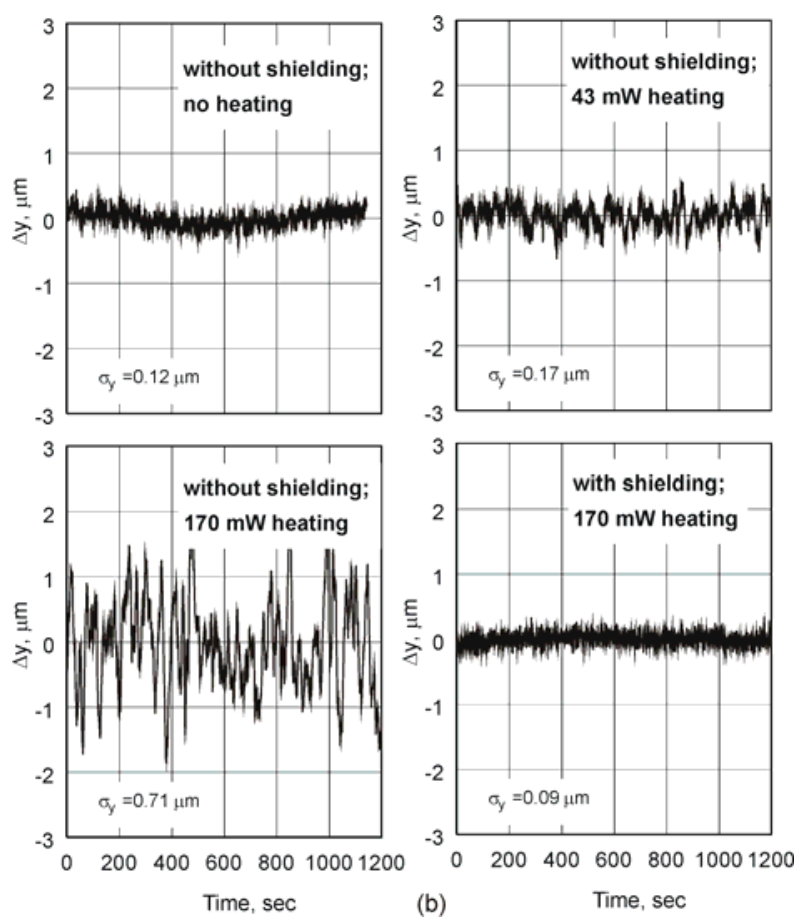

Figure 3: Temporal dependence of pointing instability (a) in the horizontal direction and (b) in the vertical direction measured at different power applied to the resistive heater. The measurements were performed at the $5 \mathrm{~Hz}$ profiler scanning frequency with averaging over 5 successive position measurements.
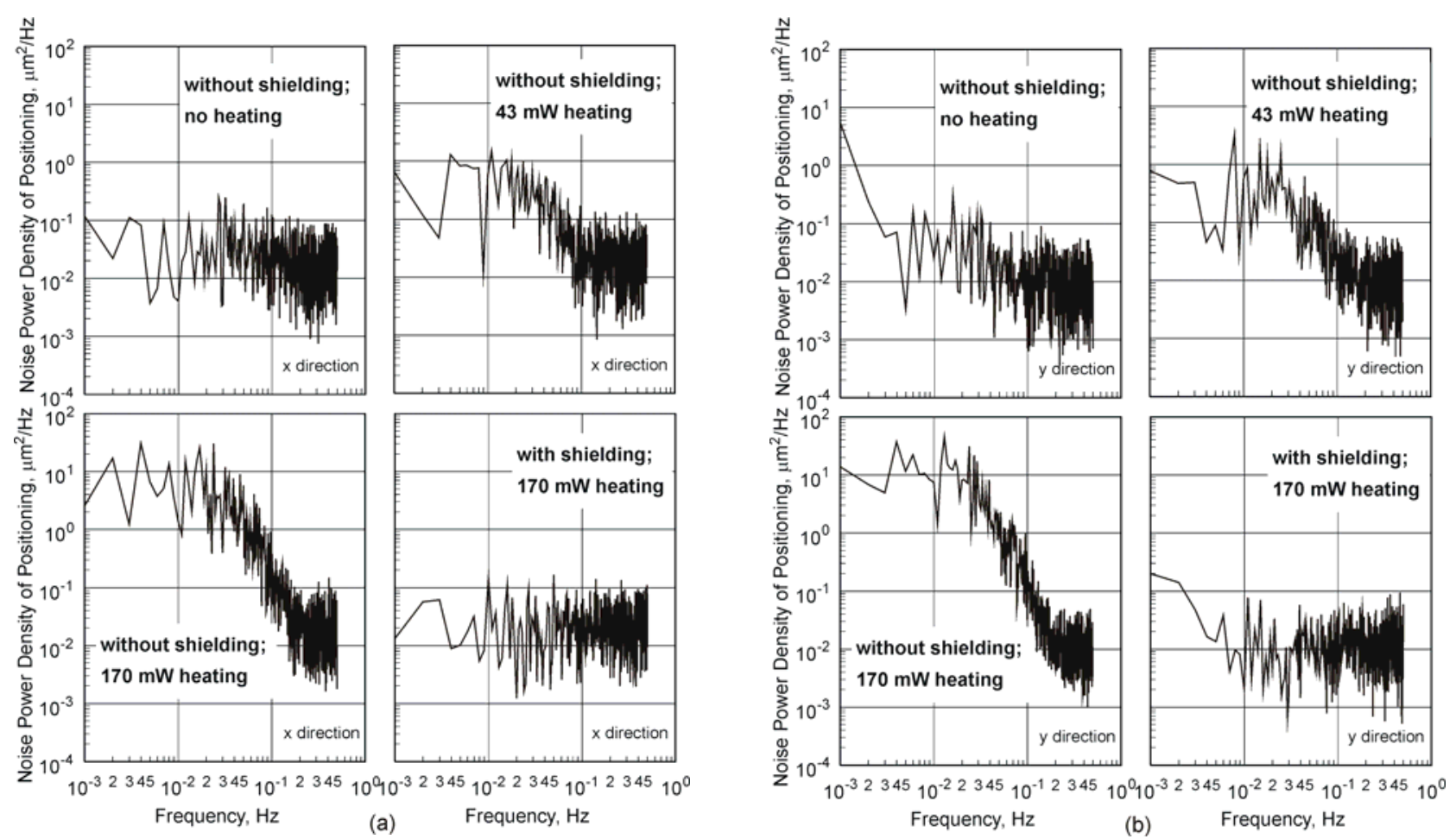

Figure 4: Noise power density spectra calculated from the data of the beam-position-variation (a) in the horizontal direction and (b) in the vertical direction shown in Fig. 1a and Fig. 1b, respectively. The lower frequency increase of the spectra obtained, when the shielding tube was removed and the heater was supplied, is due to a perturbation of the beam optical path by air convection stimulated with the heater. 

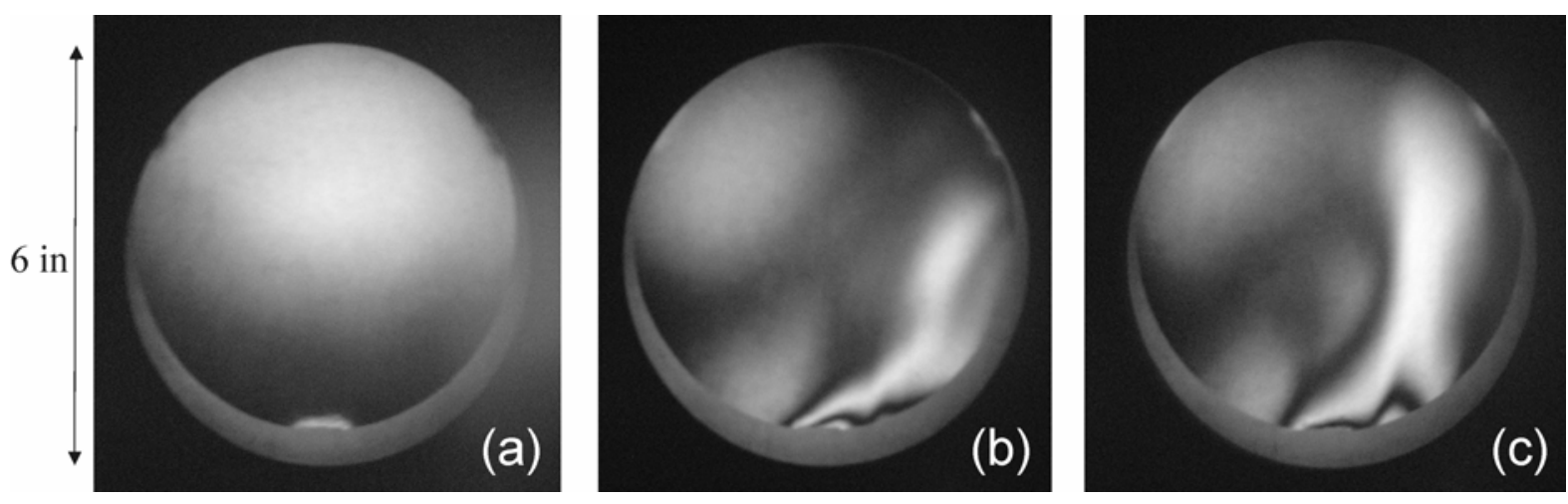

Figure 5: The interferograms obtained with the ZYGO GPI interferometer available at the OML. (a) - the image of a flat etalon with unpowered heater. (b) and (c) - the interferograms obtained when the wire heater produces approximately $1 \mathrm{~W}$ resistive heat.

The structure of the convection flow stimulated with our heater is depicted in Fig. 5. The figure shows interference pictures obtained with the ZYGO GPI interferometer. During the measurements, the heater was placed between the ZYGO output window and a flat etalon. The interferogram in Fig. 5a was recorded when the heater was not powered. Figures $5 \mathrm{~b}$ and $5 \mathrm{c}$ reproduce the interferometric images at the different times when the heater was supplied with $250 \mathrm{~mA}$ current, producing approximately $1 \mathrm{~W}$ of resistive heat. That the convection flow is rather unstable is reflected by the difference of the interferograms (b) and (c). More stable and regular interferograms looking like two upward plumes can be obtained at significantly higher power applied to a similar, wire-based, heater. ${ }^{23}$ At a higher heat power, smaller environmental perturbations do not lead to a significant change of the convective flow.

The described experiments (Figs. 3,4) suggest an approximately linear dependence of the beam position variation (rms) on the heat power applied, when the convection stimulated just with $170 \mathrm{~mW}$ power causes fluctuations of the beam position up to $\sim 1 \mu \mathrm{m}$. Taking into account the distance between the light source and the profiler (1050 mm), one can conclude about $\sim 1 \mu \mathrm{rad}$ variations of the beam direction at the $170 \mathrm{~mW}$ heater power. The NPD spectra due to air convection have a very characteristic form. In the range of frequencies from $\sim 5 \cdot 10^{-2} \mathrm{~Hz}$ to $\sim 0.5 \mathrm{~Hz}$, the spectra can be approximated with an inverse-power-law function - Fig. 4. Depending on the intensity of the convection controlled with a resistive heater, the fractal power index lies between approximately 1 and 3 .

It is interesting to mention, that spectral behavior similar to one shown in Fig. 4 has been reported for the power spectral density of the optical path difference induced by the atmosphere turbulence. ${ }^{25}$

\section{EFFECT OF AIR-BLOWING ON AIR CONVECTION NOISE IN LASER POINTING}

In the previous section it was demonstrated that the effect of air convection on laser beam pointing can be easily eliminated with a simple shielding system consisted of a metal tube surrounded the beam optical path. Similarly, a simple shield (or enclosure) over the optical head of the LTP helps to suppress air turbulence noise in the LTP sample channel. However, such an approach can not be easily applied to the LTP reference channel, where the optical path is changing in the course of a measurement. The shielding method does not also provide complete protection for a large telescope, where the atmospheric turbulence is one of the major sources for image degradation. ${ }^{26,27}$ In order to overcome the problem, adaptive optical systems compensating for wave-front corrugations have been developed and widely used for ground based telescopes. However, another much more simple method for suppressing the air convection effect is also known to be applied. ${ }^{28}$ It consists in fast agitation (blowing) of the air across the optical path.

In this section, we investigate effect of air-blowing across a laser beam optical path on beam pointing stability. The experimental arrangement is shown in Fig. 6. The air blowing system consists of 10 low-noise computer fans assembled along the 1-m long optical path. The shielding tube and the resistive heater under the tube are also seen on the picture. In the course of the experiment, the temporal beam-position measurements were carried out at different experimental arrangements: with the shield installed ('shield ON') and uninstalled ('shield OFF'); with the blowing system switched on ('fans ON') and switched off ('fans OFF').

Figure 7 presents the temporal dependences of the laser beam centroid in the horizontal direction (left-hand plots) and in the vertical direction (right-hand plots) measured with the beam profiler placed at the distance of $1050 \mathrm{~mm}$ from the laser collimator (Fig. 6). The corresponding NPD spectra are shown in Fig.8. 


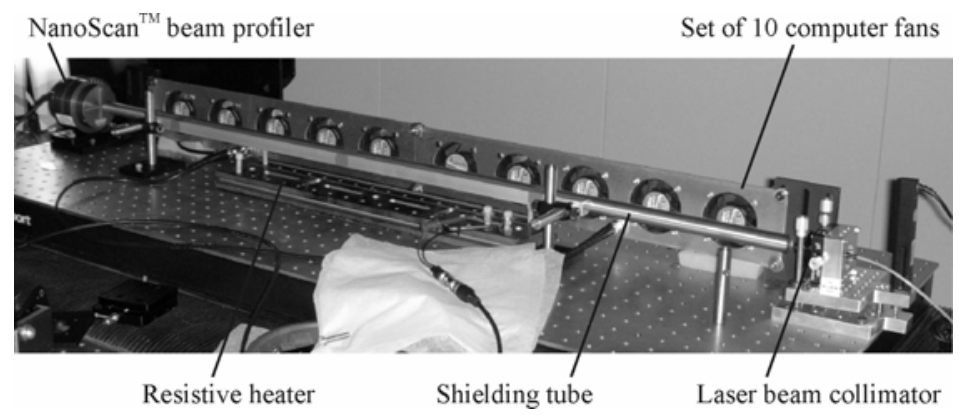

Figure 6: Experimental arrangement used for investigation of air-blowing effects on laser pointing stability. The air blowing system consists of 10 low-noise computer fans assembled along the 1050-mm long optical path. The shielding tube and the resistive heater under the tube are also seen on the picture. For more details about the experimental set-up see also text in Sec. 2.
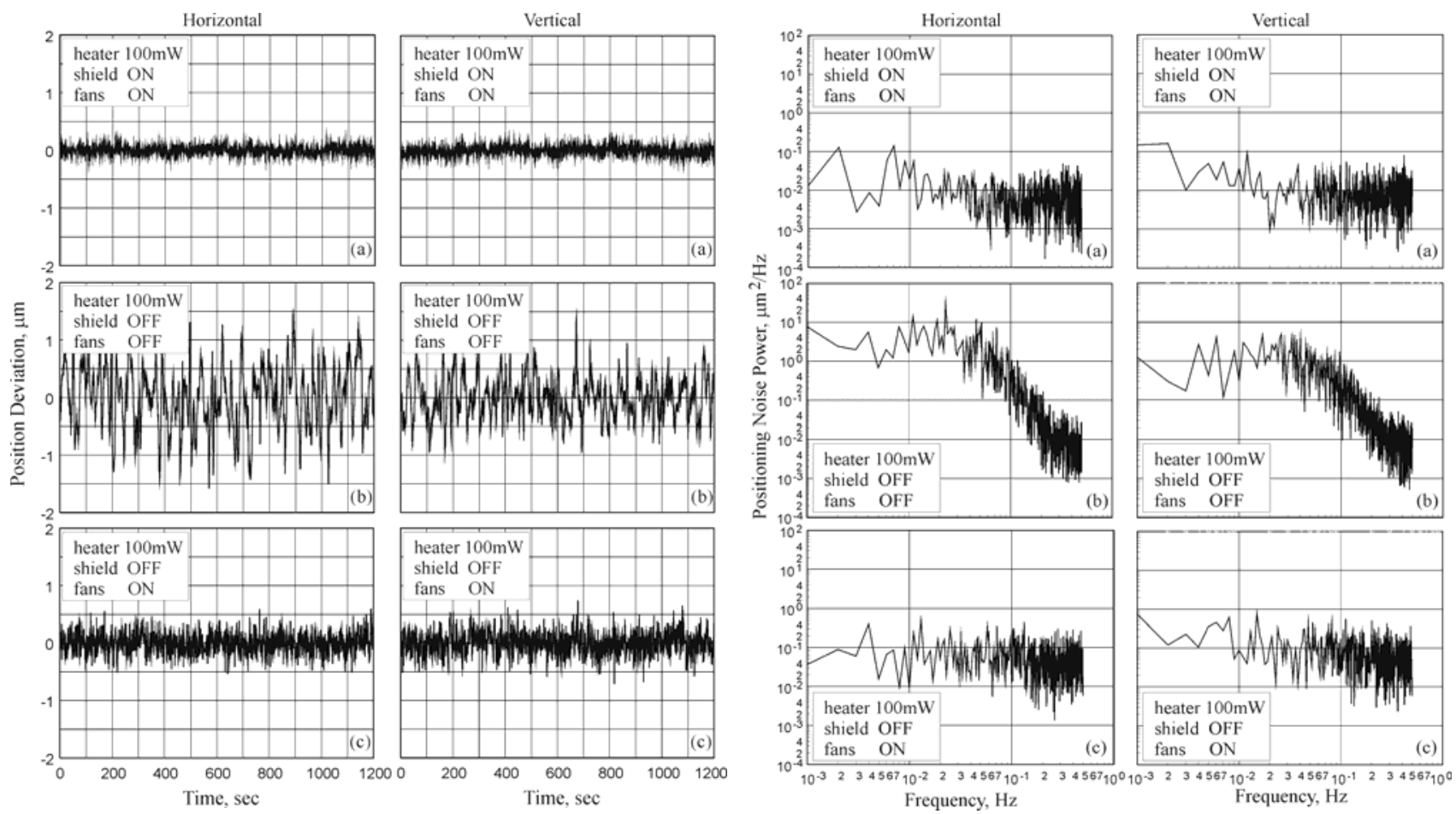

Figure 7: Temporal variation of laser beam position measured with the profiler at $1050 \mathrm{~mm}$ from the collimator. The left-hand and right-hand plots show the beam position deviations in the horizontal and in vertical direction, respectively. The measurements (a) were performed with the shielding tube installed around the light-beam optical path to shield against air convection stimulated with $100 \mathrm{~mW}$ resistive heat power. The fans were supplied with $5 \mathrm{~V}$ DC voltage. The traces (b) were obtained when the shielding tube was removed and the fans were switched off. Switching the fans on to $5 \mathrm{~V}$ leads to a significant suppression of the convection noise - traces (c). The data were measured at the profiler frequency of $10 \mathrm{~Hz}$ and with averaging over 10 profiles (time constant of $1 \mathrm{sec}$ ).

Figure 8: Noise power density spectra calculated from the data of the beam-position variations shown in Fig. 7. The lefthand and right-hand plots show the NPD spectra of the beam position deviations in the horizontal and in vertical direction, respectively. With the shielding tube installed around the light-beam optical path [plots (a)], the NPD spectra have just a tiny low-frequency deviation from a white-noise character. When the shielding tube was removed and the fans were switched off, the NPD spectra obtain a characteristic inversepower-law shape for frequencies between $\sim 5 \cdot 10^{-2} \mathrm{~Hz}$ and $\sim 0.5 \mathrm{~Hz}$. Switching the fans on to $5 \mathrm{~V}$ leads to significant suppression and randomization of the convection noise; see spectra (c).

The traces (a) in Figs. 7,8 were obtained when the shielding tube was mounted on the beam to shield the optical path from air convection stimulated with $100 \mathrm{~mW}$ resistive heat power. The fans connected in parallel were supplied with $5 \mathrm{~V}$ from a stabilized power supply. The pointing instability (rms) measured in this case to be about $0.08 \mu \mathrm{rad}$, is equal to the performance limit of the present set-up. Therefore, the shielding against the convection is reasonably efficient and the vibrations of the fans are insignificant. The traces (b) in Figs. 7,8 were obtained when the shielding tube was removed and the fans were switched off. The pointing instability (rms) corresponding to air convection stimulated with $100 \mathrm{~mW}$ 
was found to be about $0.5 \mu \mathrm{rad}$. The traces (c) were obtained with an experimental arrangement similar to (b) but with the fans switched on to $5 \mathrm{~V}$. The air-blowing suppresses the beam pointing noise by factor of approximately 2.5 , from $0.5 \mu \mathrm{rad}$ [traces (b)] to approximately $0.2 \mu \mathrm{rad}$ [traces (c)]. The data in Fig. 2 were measured at a 1 -sec time constant with a profiler frequency of $10 \mathrm{~Hz}$ and averaging over 10 profiles.

The beam pointing instability data similar to ones shown in Figs. 7 and 8 but obtained at $150 \mathrm{~mW}$ heater power are collected in Figs. 9 and 10. In this case, the suppression is even larger, from $\sim 0.8 \mu \mathrm{rad}$ [traces (a)] to approximately $0.26 \mu \mathrm{rad}$ [traces (b)], corresponding to a overall suppression factor of $\sim 3$. The noticeable observation from the data presented in Figs. 7-10 is the randomization of the convection noise under air blowing with the PC fans.

The NPD spectra at higher frequency range are shown in Fig.11. Figure 11a presents the data obtained with the heater and the fans switched off, and no shielding tube installed. In spite of the fact that before the measurement the set-up was at quiet environmental conditions overnight, the noise spectrum still has a perturbation characteristic of air convection noise seen as an increase toward lower frequencies. The spectrum in Fig. 11b differs from the one shown in Fig. 11a since the fans were switched on at $\mathrm{V}_{\mathrm{f}}=5 \mathrm{~V}$. The air blowing stimulated by the fans leads to the white-noise-like spectra, washing out the convection effect and increasing the overall level of noise by factor approximately 2 . The corresponding pointing instability (rms) is increased from $0.09 \mu \mathrm{rad}$ to $0.17 \mu \mathrm{rad}$ for lower frequency spectra (with averaging), and from $0.24 \mu \mathrm{rad}$ to $0.44 \mu \mathrm{rad}$ for higher frequency spectra (without averaging).

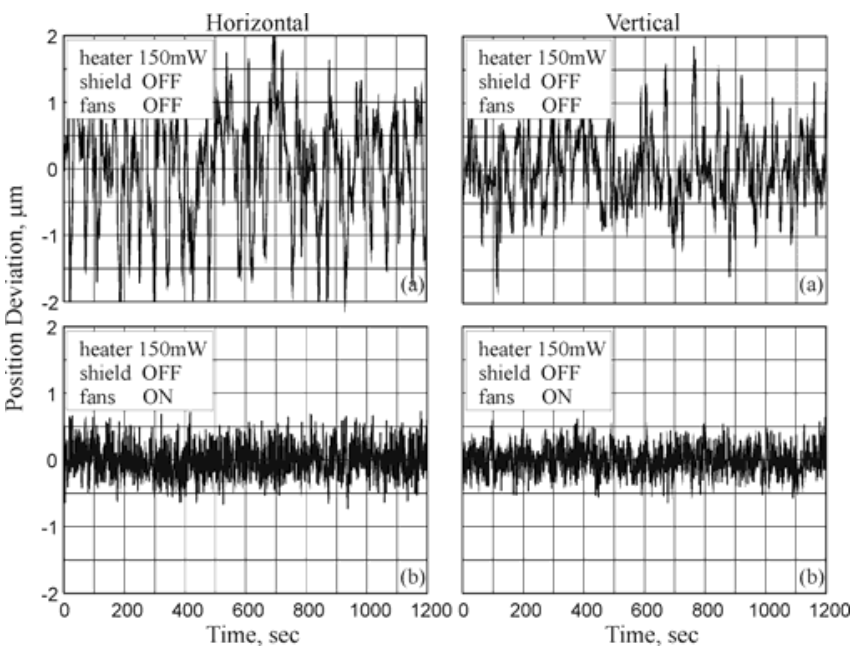

Figure 9: Temporal variation of laser beam position measured with the profiler at $1050 \mathrm{~mm}$ from the collimator at $150 \mathrm{~mW}$ power supplied to the heater. All other setting and notations are similar to that of Fig. 7.

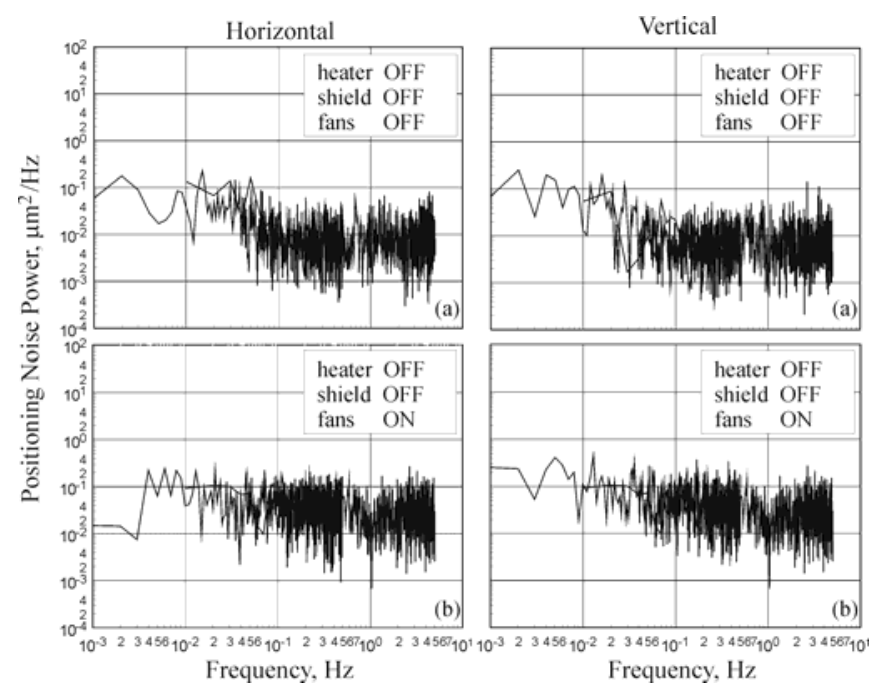

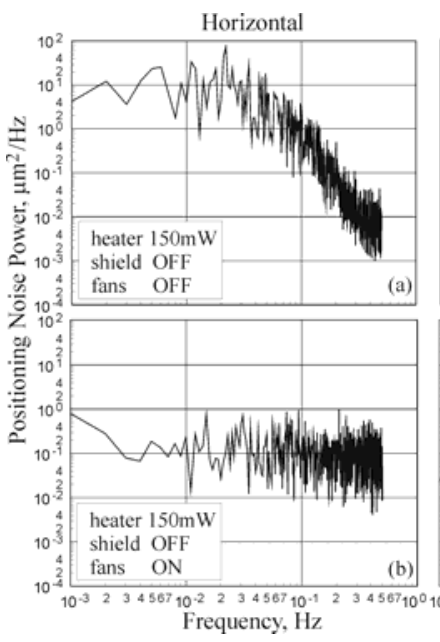

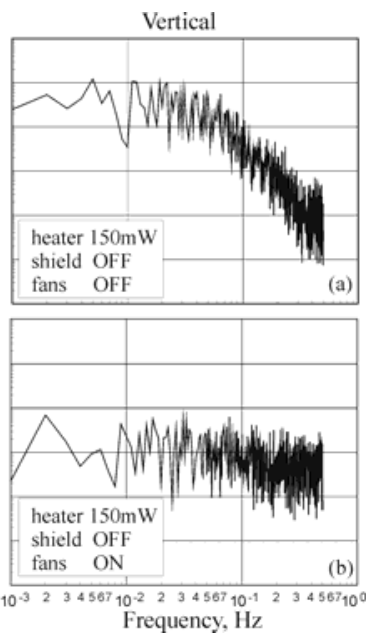

Figure 10: Noise power density spectra calculated from the data of the beam-position-variations shown in Fig. 9 and measured at $150 \mathrm{~mW}$ heater power. All other setting and notations are similar to that of Fig. 8.

Figure 11: NPD spectra measured over a larger frequency range. The spectra calculated with and without averaging over 10 measured profiles are plotted together. The left-hand and right-hand plots show the NPD spectra of the beam position deviations in the horizontal and in vertical direction, respectively. The data were obtained with the heater switched off and no shielding tube installed. (a) The fans are off; (b) the fans are supplied with $5 \mathrm{~V}$. 
The air-blowing effect on the beam pointing stability was also investigated as a dependence on the voltage $\mathrm{V}_{\mathrm{f}}$ applied to the fans in the range of $4 \mathrm{~V} \leq \mathrm{V}_{\mathrm{f}} \leq 7 \mathrm{~V}$. It was found that at $\mathrm{V}_{\mathrm{f}}=4 \mathrm{~V}$, the air blowing is not efficient enough in order to fully suppress low frequency noise due to convection stimulated with $150 \mathrm{~mW}$ power. From other side at $\mathrm{V}_{\mathrm{f}}=7 \mathrm{~V}$, the high frequency noise is noticeably increased. The empirical optimum is nearby $\mathrm{V}_{\mathrm{f}}=5 \mathrm{~V}$ used throughout the work.

\section{AIR CONVECTION AND PSD MEASUREMENTS WITH THE LTP}

The observed spectral dependences (Figs. 11 and 12) of the beam positioning noise due to air convection have a fractallike (inverse-power-law) behavior in the range of frequencies from $\sim 5 \cdot 10^{-2} \mathrm{~Hz}$ to $\sim 0.5 \mathrm{~Hz}$. Depending on the intensity of the convection controlled with the heater power, the fractal power index lies between $\sim 2(43 \mathrm{~mW})$ and $\sim 3(170 \mathrm{~mW})$. At lower frequencies, the spectra have a white spectral noise character. Such a behavior of low frequency noise in convective systems was analyzed in Ref. ${ }^{29}$ (see also references therein).

A similar behavior of the power spectral density distributions measured with the LTP was recently observed with a gold coated stainless steel mirror. ${ }^{19}$ The similarity of the NPD and PSD spectra noticed in Ref. [2] can be explained assuming that the LTP measurements are significantly affected by the air convection along the optical paths of the sample and the reference beams.

Figure 12a presents the PSD spectrum of a stainless steel mirror measured with the LTP. ${ }^{19}$ In the course of the measurement, the LTP carriage was translated along the mirror with speed of $2 \mathrm{~mm} / \mathrm{sec}$ and the sampling distance was $1 \mathrm{~mm}$. The total measured trace was $340 \mathrm{~mm}$. Therefore, the measurement covers a frequency range from $\sim 6 \cdot 10^{-3} \mathrm{~Hz}$ to $\sim 1 \mathrm{~Hz}$. Note that in this case, the optical path for each of the LTP channels (reference and sample) has a meter scale. In order to compare the result of this measurement with the noise spectra characteristic for the air convection, the PSD distribution was re-evaluated as a function of the scanning frequency. The result is shown in Fig. 12b.

Note that there is a simple rule how to recalculate the power index of an inverse-power-law approximation of a PSD spectrum of a surface height variation to the power index of the corresponding noise spectrum presented in the surface slope domain. The derivative theorem of the Fourier transform (see, e.g. ${ }^{14}$ ) states that if $F(u)$ is a Fourier transform of function $h(x)$, than the derivative $h^{\prime}(x) \equiv \alpha(x)$ has the Fourier transform $i 2 \pi u F(u)$. Therefore, if the PSD of a surface height variation $h(x)$ is approximated with an inverse-power-law function $S_{h}(u)=S(1) / u^{B}$, then the PSD of the slope variation $\alpha(x) \equiv h^{\prime}(x)$ has to be described with a power-law function $S_{\alpha}(u)=4 \pi^{2} u^{2} S(1) / u^{B} \propto 1 / u^{B-2}$, where $u$ is spatial frequency variable. That suggests that the slope power index is smaller by 2 than the power index found from the surface height variation.
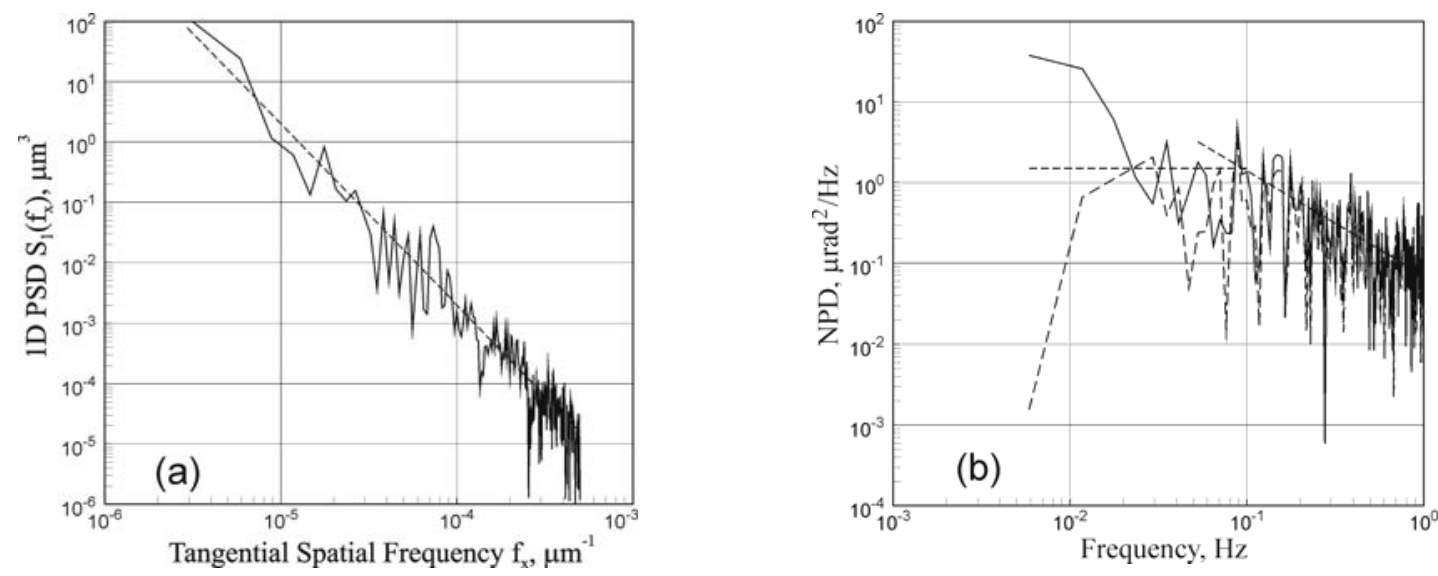

Figure 12: (a) One dimensional (1D) PSD spectrum a stainless steel mirror measured with the LTP. (b) The same PSD measurement presented as a dependence of the slope NPD on sampling frequency. The dashed line in plot (a) shows a fractal approximation with a power index equal to 3. The dashed lines in plot (b) depict the characteristic behavior of the NPD spectra due to air convection: a linear slope at higher frequencies and a white-spectral noise at lower frequencies.

In Fig. 12b, dashed lines depict the characteristic behavior of the NPD spectra due to air convection: a linear (in log-log scale) slope at higher frequencies and a white-spectral noise at lower frequencies (compare with Figs. 4, 8 and 10). The solid and long-dashed traces in Fig. 12b differ in the detrending procedure applied to the measured slope trace. The solid trace was obtained with a first-order polynomial detrending. The long-dashed trace is after detrending with a 
fourth-order polynomial, when the mirror figure due to a support system is extracted. One can see that detrending perturbs just a couple of the lower-frequency points.

Thus, there is an obvious similarity of the spectra in Figs. 4, 8 and 10 measured when the convection is stimulated and the LTP data shown in Fig. 12b. The similarity suggests that power PSD measurements with the LTP could be significantly affected by air convection noise and, in such a situation, should be cautiously used as a measure of a mirror's slope and figure.

\section{AIR CONVECTION NOISE IN THE LTP REFERENCE CHANNEL}

Figure 13 shows a set-up used for an experiment to compare the slope noise signals recorded in the reference channel of the LTP at an air-blowing system switched off and switched on. The computer-fan-based air blowing system described above was split into two sets, each of five fans. The fans of the horizontal set are uniformly distributed along the optical path between the LTP optical head and a reference mirror (Fig. 13). The vertical set is mounted along the optical path created with the LTP folding mirrors, which are the parts of the optical head.

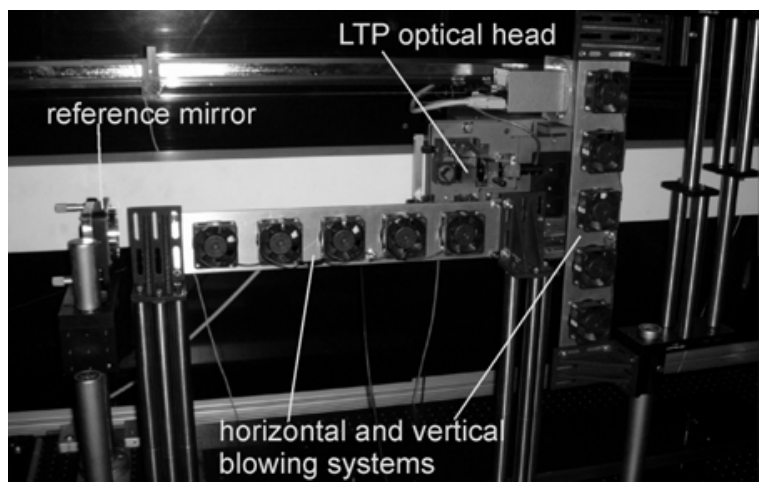

Figure 13: Set-up for measurement of air convection noise in the LTP reference channel.

Figures $14 \mathrm{a}$ and $15 \mathrm{a}$ present the temporal dependences of a slope variation in the LTP reference channel recorded with the blowing system switched off and switched on, respectively. The measurements were performed in the LTP II self-test mode. In this mode, the carriage with optical head does not move and, therefore, the effects related to the carriage wobbling and wiggling while moving are excluded. Each trace in Figs. 14 and 15 consists of 1000 points measured with $0.5 \mathrm{sec}$ interval Such a regime is characteristic of the LTP measurement of a significantly long, about $0.5-1$ meter long, mirror.

An obvious result of the air blowing seen in Fig. 15a is a significant increase of the higher frequency noise. However, at air-blowing on, the overall noise variation (rms) was even slightly deceased from approximately $0.95 \mu \mathrm{rad}$ to $0.8 \mu \mathrm{rad}$.

In order to illustrate a decrease of noise at lower frequencies, the data shown in Figs. 14a and 15a was filtered with averaging over 10 sequential points. The results of filtering are presented in Figs. 14b and 15b, respectively. After filtering, the rms variation of the trace measured at the blowing system off was not noticeably changed, while the rms variation of the trace measured at the blowing system on was decreased by factor of two, reaching approximately $0.41 \mu \mathrm{rad}$. Moreover, peak-to-valley variation was also decreased by factor of two.

\section{CONCLUSION}

In the present work, we have investigated the effect of air convection on laser-beam pointing noise described with noise power density frequency distributions. It has been shown that the NPD spectra due to air convection have a very characteristic form. In the range of frequencies from $\sim 0.05 \mathrm{~Hz}$ to $\sim 0.5 \mathrm{~Hz}$, the spectra can be modeled with an inversepower-law function. Depending on the intensity of air convection that is controlled with a resistive heater of 40 to 170 $\mathrm{mW}$ along a one-meter-long optical path, the power index lies between 1 and 3 at the overall rms noise from $\sim 0.2$ to $\sim 1 \mu \mathrm{rad}$. 

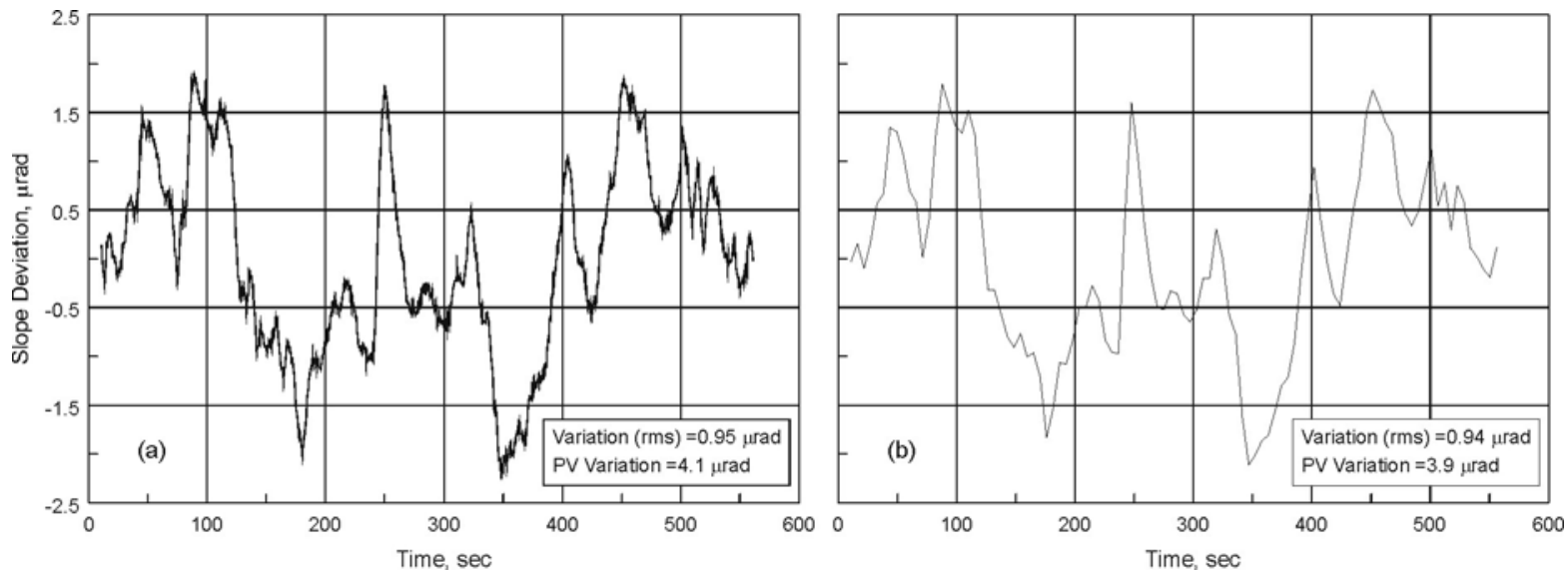

Figure 14: a - Temporal dependence of the slope deviation signal measured in the LTP reference channel with the blowing system switched off; $b$ - the same measurement filtered with averaging over 10 sequential points.
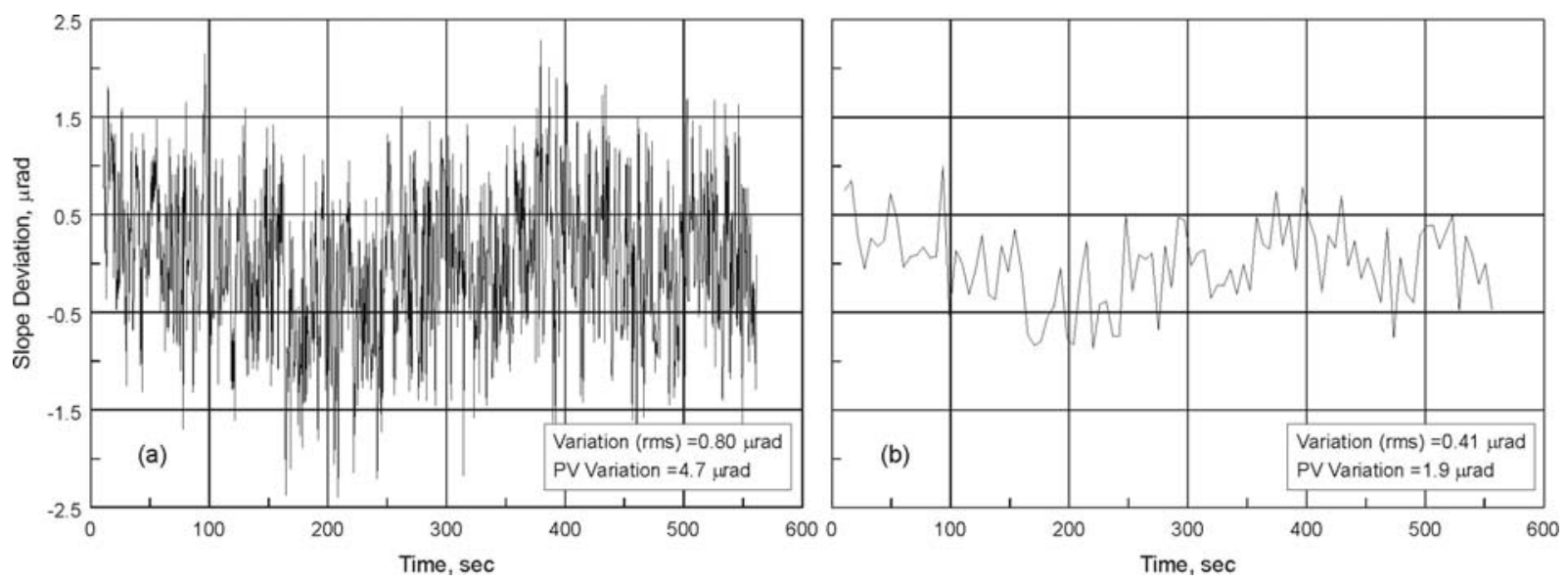

Figure 15: a - Temporal dependence of the slope deviation signal measured in the LTP reference channel with the blowing system switched on; $b$ - the same measurement filtered with averaging over 10 sequential points.

The performed experiments have shown a high efficiency of the developed air-blowing technique based of a set of 10 PC fans to suppress the low frequency noise of the laser beam pointing induced by air convection along a one-meter-long optical path. Air-blowing stimulated this way leads to a white-noise-like spectrum. The spectrum is a result of a tradeoff, when the fans are turned on the noise in the low frequency part of the spectrum goes down at the expense of an increase in the high frequency end of the spectrum.

Similar behavior of power spectral density distributions measured with the LTP was recently observed in Ref. ${ }^{19}$ It was demonstrated here that the similarity is due the effect of air convection on LTP measurements. The ability to change with air-blowing the spectral characteristics of the air turbulence on the beam pointing stability allowed us to investigate the contribution of this noise source to the LTP reference channel.

The performed experiments have demonstrated a significant perturbation of the LTP measurements due to air convection effects. It has been also shown that the air-blowing technique developed and based on PC fans allows a significant suppression of the noise at lower frequencies. The experiments were performed with the present (original) arrangement of the LTP-II, which uses a simple diode laser without temperature stabilization. At quiet environmental conditions inside a hutch, the pointing instability of the laser has been characterized with an rms variation of approximately $0.36 \mu \mathrm{rad}$. Such a variation is comparable with the rms variation of the filtered reference signal $(0.41 \mu \mathrm{rad})$ recorded with air-blowing on. 
Note that the higher frequency noise measured with the LTP with air-blowing on (Fig. 15) significantly exceeds the noise of $0.17 \mu \mathrm{rad}(\mathrm{rms})$ observed with a fiber-coupled diode laser system and a Nano-scan beam profiler (Fig.11). The difference can probably be attributed to the strong temperature dependence of the LTP laser pointing exhibited, while an intensive air blowing across the laser is applied. In order to effectively suppress the air convection noise in a new version of the LTP, one should use a more sophisticated light source e.g., one based on a fiber coupling. The pointing stability of different lasers has also been investigated in Ref. ${ }^{30}$

\section{ACKNOWLEDGEMENTS}

The authors are grateful to E. Church, H. Padmore and T. Warwick for extremely useful discussions. This work was supported by the U. S. Department of Energy under contract number DE-AC02-05CH11231.

\section{DISCLAIMER}

Certain commercial equipment, instruments, or materials are identified in this document. Such identification does not imply recommendation or endorsement by the US Department of Energy, LBNL or ALS nor does it imply that the products identified are necessarily the best available for the purpose.

\section{REFERENCES}

1. E. L. Church, P. Z. Takacs, Use of an optical profiling instrument for the measurement of the figure and finish of optical quality surfaces, Wear, 109 (1986), 241-57.

2. P. Z. Takacs, Shinan Qian, J. Colbert, Design of a long trace surface profiler, Proceedings of SPIE 749 (1987), 5964.

3. P. Z. Takacs, S. K. Feng, E. L. Church, Shinan Qian, W-M. Liu, Long trace profile measurements on cylindrical aspheres, Proceedings of SPIE, 966 (1989), 354-64.

4. S. C. Irick. W. R. McKinney, Advancements in one-dimensional profiling with a long trace profiler, Proceedings of SPIE, 1720 (1992), 162-8.

5. S. C. Irick, W. R. McKinney, D. L. Lunt, P. Z. Takacs, Using a straightness reference in obtaining more accurate surface profiles from a long trace profiler (for synchrotron optics), Rev. Sci. Instrum., 63(1), 1436-8 (1992).

6. S. C. Irick, Improved measurement accuracy in a long trace profiler: compensation for laser pointing instability, Nuclear Instruments \& Methods in Physics Research A-Accelerators Spectrometers Detectors \& Associated Equipment, 347(1-3), 226-30 (1994).

7. P. Z. Takacs, Shinan Qian, T. Kester, H. Li, Large-mirror figure measurement by optical profilometry techniques, Proceedings of SPIE, 3782 (1999), 266-74.

8. Shinan Qian, G. Sostero, P. Z. Takacs, Precision calibration and systematic error reduction in the long trace profiler, Optical Engineering, 39(1) (2000), 304-10.

9. P. Z. Takacs, Shinan Qian, Accuracy limitations in long-trace profilometry, AIP Conference Proceedings, 708 (2004), 831-4.

10. P. Z. Takacs, E. L. Church, C. J. Bresloff, L. Assoufid, Improvements in the accuracy and the repeatability of long trace profiler measurements, Applied Optics, 38(25) (1999), 5468-79.

11. V. V. Yashchuk, S. C. Irick, A. A. MacDowell, Elimination of 'ghost'-effect-related systematic errors in metrology of X-ray optics with a long trace profiler, Proceedings of SPIE 5858-34 (2005).

12. J. Pawliszyn, M. F. Weber, M. J. Dignam, Dual-beam laser deflection sensor, Rev. Sci. Instrum. 56(9), 1740-3 (1985).

13. M. C. Geckini and D. Yavus, Discrete Fourier Transformation and Its Applications to Power Spectra Estimation (Elsevier scie. Publ. Co., Amsterdam, 1983).

14. R. N. Bracewell, The Fourier Transform and Its Applications (Tata McGraw-Hill Publ. Co. Ltd., New York, 2003).

15. P. Horowitz and W. Hill, The Art of Electronics (Cambridge Univ. Press, New York, 1994).

16. V. V. Yashchuk, S. C. Irick, E. M. Gullikson, M. R. Howells, A. A. MacDowell, W. R. McKinney, F. Salmassi, T. Warwick, "Crosscheck of different techniques for two dimensional power spectral density measurements of X-ray optics," in Advances in Metrology for X-Ray and EUV Optics, L. Assoufid, P. Z. Takacs, J. S. Taylor, eds., Proc. SPIE 5921, 105-116 (2005).

17. J. C. Stover, Optical Scattering: Measurements and Analysis (SPIE, Washington, 1995). 
18. V. V. Yashchuk, A. D. Franck, S. C. Irick, M. R. Howells, A. A. MacDowell, W. R. McKinney, "Two dimensional power spectral density measurements of X-ray optics with the Micromap interferometric microscope," in Nano- and Micro-Metrology, H. Ottevaere, P. DeWolf, D. S. Wiersma, eds., Proc. SPIE 5858, 85-96 (2005).

19. Valeriy V. Yashchuk, Eric M. Gullikson, Malcolm R. Howells, Steve C. Irick, Alastair A. MacDowell, Wayne R. McKinney, Farhad Salmassi, Tony Warwick, James P. Metz and Thomas W. Tonnessen, Surface Roughness of Stainless Steel Bender Mirrors for Focusing Soft X-rays, Applied Optics, 45(20) (2006), 4833-42.

20. Photon Inc., http://photon-inc.com.

21. Melles Griot Inc., http://lasers.mellesgriot.com.

22. W. H. Press, S. A. Teukolsky, W. T. Vetterling, B. P. Flannery, Numerical Recipes in C++: The Art of Scientific Computing (Second Ed., Cambrige Univ. Press, Cambridge, 2003).

23. D. W. Hatfield and D. K. Edwards, Edge and Aspect Ratio Effects on Natural Convection from the Horizontal Heated Plate facing downwards, Int. Journal of Heat Mass Transfer 24(6), 1019-24 (1981).

24. L. Pera and B. Gebhard, Laminar Plume Interactions, Journal of Fluid Mechanics 88(2), 461-463 (1975).

25. R. P. Linfield, M. M. Colavita, and B. F. Lane, Atmospheric turbulence measurements with thePalomar Testbed Interferometer, The Astronomical Journal 554 (1-1), 505-513 (2001).

26. F. Roddier, The effects of atmospheric turbulence in optical astronomy, in Progress in Optics, E. Wolf ed, Vol. XIX, 281-376 (1981).

27. E. Di Folco, B. Koehler, P. Kervella, M. Sarazin, V. C. du Foresto, M. Scholler, M. Wittkowski, Atmospheric and internal turbulence measured on the very large telescope interferometer with VINCI, Proceedings of SPIE 4838, 1115-26 (2003).

28. The United Kingdom Infrared Telescope, http://outreach.jach.hawaii.edu/articles/moreaboutukirt.

29. S. Zaleski, Low-frequency noise from random dislocation motion in large convective system, Phys. Rev. A 39(6), 3088-3093 (1989).

30. J. Gray, P. Thomas, and X. D. Zhu, Laser pointing stability measured by an oblique-incidence optical transmittance difference technique, Review Scientific Instruments 72(9), 3714-3717 (2001). 